\title{
Variability and Spectra of AGN in the EUV and the Relation to Other Bands
}

\author{
HERMAN L. MARSHALL \\ Eureka Scientific, Inc., 5 Whipple Rd., Lexington, MA, 02173 USA
}

\begin{abstract}
Data from several collaborations will be shown which demonstrate the utility of $E U V E$ observations. For Mk 478, a Seyfert 1 galaxy, the rapidly variable EUV flux is shown to have a steep, featureless continuum. The EUV data are combined with UV and optical data to form an overall spectrum that is consistent with an accretion disk model; slight temperature variations in the innermost regions could cause the large EUV flux changes. EUVE data for other sources are presented: NGC 5548, which shows significant variations and has an EUV spectrum that shows no emission lines, contrary to a previous report; $3 \mathrm{C} 273$, which did not vary much; and PKS 2155-304, which was observed simultaneously with $A S C A$ and $I U E$ when a hard X-ray flare was detected as an EUV polarization measurement was being attempted.
\end{abstract}

There were 13 AGN reported by Marshall et al. (1995a) that were detected in the $E U V E$ all-sky survey. Of these, 6 have now been observed with the $E U V E$ spectrometer and 4 of these observations will be discussed here.

\section{Mrk 478}

The observations of this source are reported in more detail by Marshall et al. (1995b). Figure 1 shows the light curve in the deep survey (DS) imaging detector, which obtains a direct image of the target during spectroscopic observations. The light curve shows variability of a factor of $2-10$ over time scales of less than one day.

The spectrum shows no emission lines and is consistent with a very steep power law spectrum, with an energy index $\alpha=4.70 \pm 0.65$ if the column density is fixed at the newly measured Galactic value, $\log N_{\mathbf{H}}=20.0$. Combining these data with (nonsimultaneous) UV and optical data indicate that a significant fraction of the total luminosity may be in the EUV band and appears to fit an accretion disk spectrum, such as modelled by Sun \& Malkan (1989). The fit gave a central black hole mass of $1.3 \times 10^{8} M_{\odot}$ and an accretion rate of $0.3 M_{\odot} / \mathrm{yr}$ and requires a highly inclined disk so that the Eddington limit not be violated.

The smooth spectrum and rapid variability can be rationalized in a model where the EUV flux derives from the inner edge of an accretion disk where the peak temperature, $T$, is about $2.5 \times 10^{5} \mathrm{~K}$. The EUV flux varies as $T^{8.2}$ because the bandpass is just over the black body peak and in the Wien tail of the spectrum. Thus a $10 \%$ change in the temperature would give a $\times 2$ change in the EUV count rate but only a $10 \%$ change in the optical flux.

\section{NGC 5548}

Observations of this source will be reported in more detail by Marshall et al. (1995c). The light curve is shown in Figure 2 and shows variations of a factor of 2 in less than 2 days at TJD $(\equiv \mathrm{JD}-2440000)=9062.5$ and 9107.5 . Even with simultaneous UV coverage, however, we could not discern the relationship to the UV band or the emission lines unambiguously. The data are consistent with zero lag or a 5 day lag (EUV leading) due to the sparse sampling of the UV data (only once per day). 


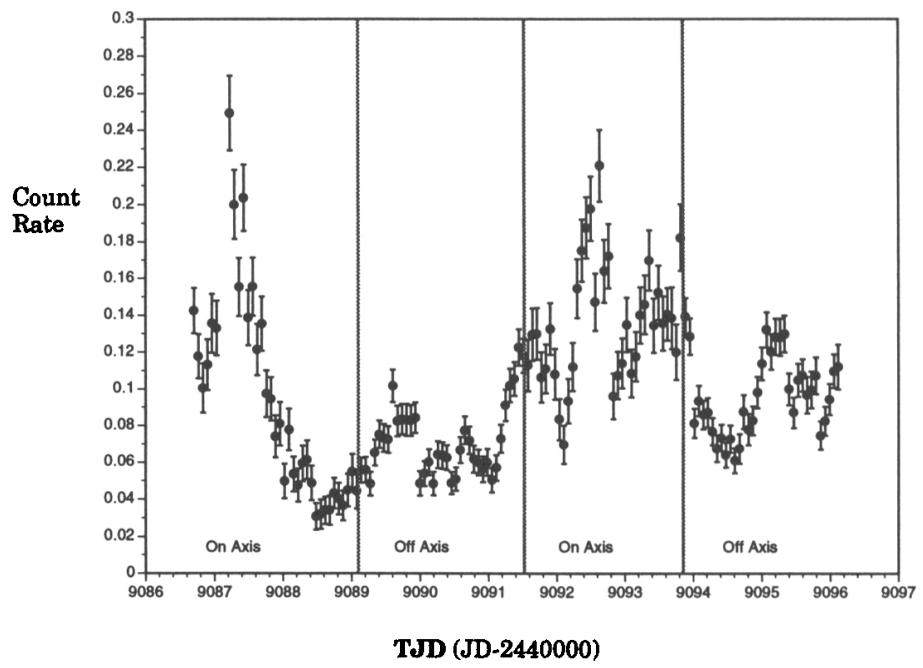

FigurE 1. Time history of the Mrk 478 Deep Survey (DS) count rates (counts $s^{-1}$ ). Note the significant variations in less than 1 day. On-axis data have systematic uncertainties due to the deadspot correction, described by Marshall et al. (1995b).

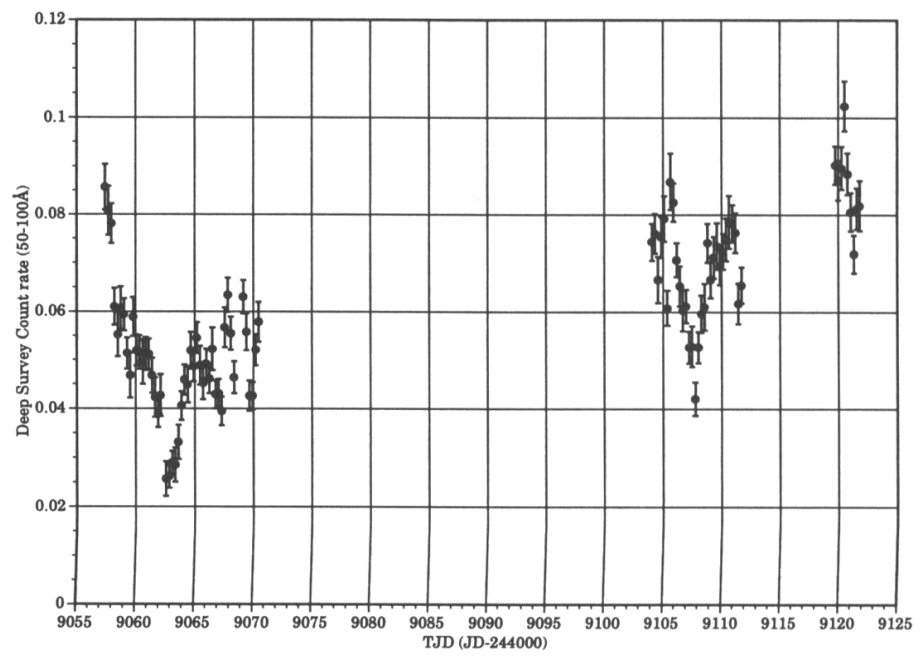

FIGURE 2. Time history of the NGC 5548 DS count rates (counts $\mathrm{s}^{-1}$ ). Note the significant variations in less than 1 day. The dead spot was no factor in this analysis since the source was observed 0.3 degrees off-axis. 


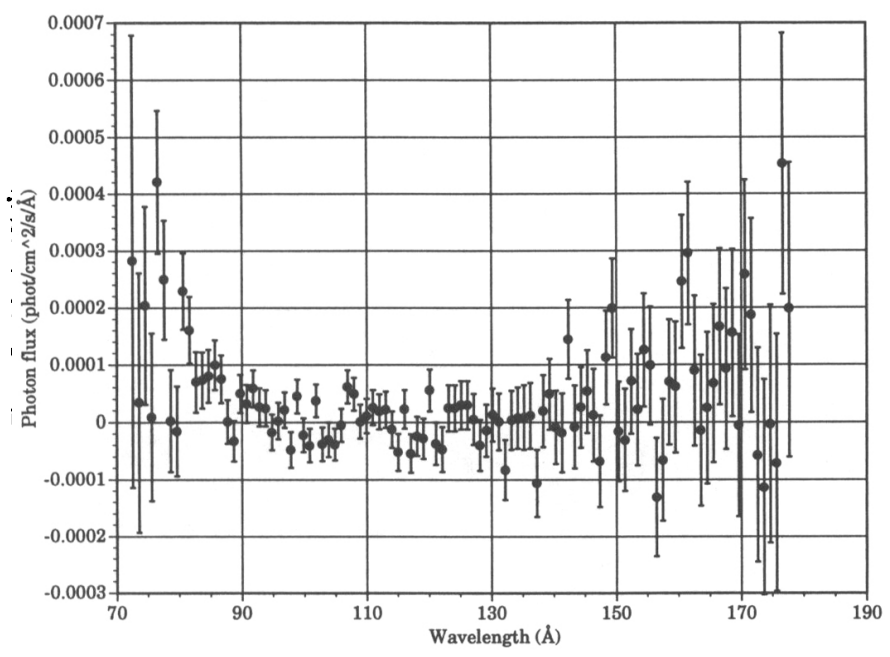

Figure 3. The EUV spectrum of 3C 273 , taken in January, 1994. The EUV flux is lower than for Mrk 478, so the spectrum is not as easy to detect, although there is significant flux in the 75-90 $\AA$ region. Again, there are no apparent emission lines.

Contrary to the report by Kaastra et al. (1995), we find no emission lines in the EUV spectrum. Specifically, our data reduction shows no detectable emission in the $1 \AA$ band centered at $90 \AA$ where the strongest line reported by Kaastra et al. (1995) was. The spectrum appears to be strongly cut off by cold matter absorption so the DS data must be dominated by continuum or emission lines in the 50-70 $\AA$ region. Our reconciliation of these results involves the details of the background, which has a faint systematic ripple (probably due to microchannel plate fiber bundles) that is difficult to remove. An excess variance results from this background variation as well.

\section{3C 273}

There is only weak evidence for variations in the DS count rate during the 6 day observation of $3 \mathrm{C} 273$ in January, 1994. The count rate was nearly constant at 0.09 count/s but dipped for one day to 0.07 count/s. A preliminary spectrum is shown in Figure 3; the signal is very low but is detectable in the $75-90 \AA$ region. Again, there are no obvious emission lines in the EUV spectrum, so the emission could originate in an accretion disk or from Comptonization of disk photons.

\section{PKS 2155-304}

The objective of the 9 day observation of PKS 2155-304 was to measure the EUV polarization by a new technique, first described by Marshall (1994). Briefly, a polarized source should produce a slight $\cos 2 \theta$ variation of the azimuthal image intensity when observed off-axis by the DS telescope. This observation is very difficult to perform, since 


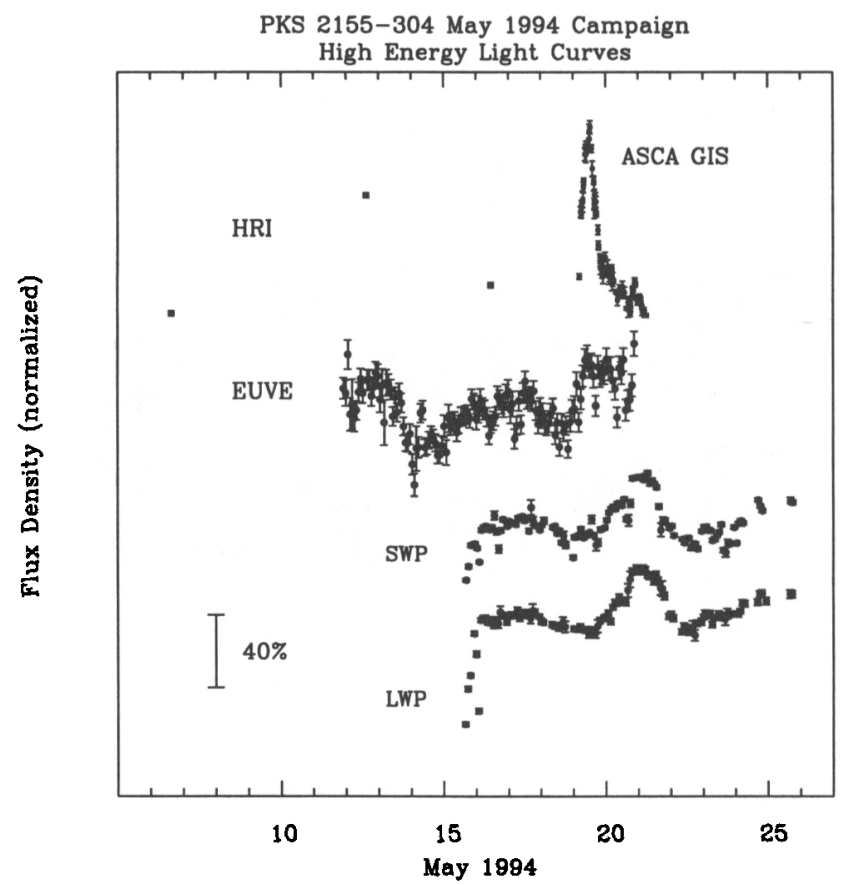

FIGURE 4. Light curves for PKS 2155-304 observed by the ASCA GIS and the ROSAT HRI (top), EUVE (middle) and the IUE SWP and LWP (bottom). The curves have been displaced for clarity. Note the UV feature on May 16 and the X-ray flare on May 19, neither of which are apparent in the EUV data.

the maximum modulation is expected to be about $4 \%$ when the source is $100 \%$ polarized. Results of analyzing these data are still in preparation.

Meanwhile, however, the light curve was generated very soon after the observation. It appeared to be uninteresting, showing a drop of $30 \%$ in 0.4 days at TJD 9486.3 and an increase of similar magnitude from TJD 9491.5 to 9492.0 . These data became significantly more interesting when combined with the $A S C A$ and $I U E$ light curves, shown in Figure 4.

There are two remarkable features in this figure, one in each of the UV and X-ray light curves and neither of these show up in the EUVE band, which overlaps them both. At TJD 9488.4 (May 15.9, 1994), both the SWP and LWP detectors on IUE registered significant increases which were not accompanied by any significant change in the EUVE count rate. The $\mathrm{X}$-ray coverage was too sparse to be of much help at this time. The second feature occurs between TJD 9491.6 and 9492.4 (on May 19, 1994), when the count rate in the $A S C A$ GIS is seen to increase by a factor of two and then return. Due to the fine data sampling in both $I U E$ and $E U V E$, we can firmly state that there is no corresponding flare in either of these two bands. At most, there may be a delayed response in the UV band lagging the X-ray band by 2 days. The case of the EUV flux is more curious; there is only the rate increase which is simultaneous with the $A S C A$ rate increase but there is no corresponding drop of the EUV flux. These observations are being prepared for publication by Urry et al. (1995). 
These observations would normally indicate that the X-ray and EUV bands are decoupled and that the UV band is only weakly coupled to either band. Previous observations (Edelson et al. 1995), however, showed a very tight correlation between these bands, so the behavior of the overall spectrum of PKS 2155-304 (and possibly BL Lac objects in general) is now in doubt. We have no explanation for the apparently disparate behaviors.

\section{Summary}

We have observed many AGN and the EUV count rates of all of them show at least $30 \%$ variability over time scales of a day. Mrk 478 shows the most dramatic variability, sometimes reaching a factor of 10 . None of them show emission lines (see Fruscione et al. 1994 for a spectrum of PKS 2155-304). Except for PKS 2155-304, the EUV flux from AGN may be due to the high energy tail of an accretion disk spectrum and the best evidence has been obtained for Mrk 478. There is still a poorly understood mismatch of the fluxes in different wavebands from PKS 2155-304-longer coverage may help resolve the discrepancy.

This work has been supported under NASA contracts NAS5-32485 and NAS5-32678 to Eureka Scientific, Inc. a private corporation created to promote the active pursuit of scientific research.

\section{REFERENCES}

Edelson, R., ET AL. 1995, Multiwavelength Monitoring of the BL Lacertae Object PKS 2155. IV. Multiwavelength Analysis, ApJ, 438, 120

Fruscione, A., Bowyer, S. Konigl, A. \& KahN, S. M. 1994, Extreme Ultraviolet Spectroscopy of PKS 2155, ApJ, 422, 55

KaAstra, J. S., Roos, N. \& Mewe, R. 1995, EUVE Observations of NGC 5548, Astr. Ap., submitted

Marshall, H. L., Carone, T. E., Shurl, J. M., Malkan, M. A., \& Elvis, M. 1994, Conceptual Design of a Fast Soft X-ray Polarimeter, Proc. SPIE, 2283, 75

Marshall, H. L., Fruscione, A., \& Carone, T. E. 1995, Active Galaxies Observed during the Extreme Ultraviolet Explorer All Sky Survey., Ap. J., 439, 90

Marshall, H. L., Carone, T. E., Shull, J. M., Malkan, M. A., \& Elvis, M. 1995, The Steep Soft X-ray Spectrum of the Highly Variable Active Nucleus in Mk 478, ApJ, submitted

MARSHALL, H. L., ET AL. 1995, in preparation

SUN, H-S., \& MalKaN, M. A. 1989, Fitting improved accretion disk models to the multiwavelength continua of quasars and active galactic nuclei, ApJ, 346, 68

URRY, C. M., ET AL. 1995, in preparation 